Pacific Journal of Mathematics

MINIMAL IC CHARACTERIZATION OF A CLASS OF
MURALS IN CONSTANT CURVATURE

G. D. JOHNS 


\title{
AN INTRINSIC CHARACTERIZATION \\ OF A CLASS OF MINIMAL SURFACES IN CONSTANT CURVATURE MANIFOLDS
}

\author{
Gene Douglas Johnson
}

\begin{abstract}
Let $X$ be an $N$-manifold of constant sectional curvature. A class of minimal surfaces in $X$, called exceptional minimal surfaces, will be defined in terms of the structure of their normal bundles. It will be shown that these surfaces can be characterized intrinsically in a way that generalizes the Ricci condition for minimal surfaces in Euclidean 3-space. It will also be shown that these surfaces are rigid when $N$ is even and belong to 1-parameter families of isometric surfaces when $N$ is odd.
\end{abstract}

0. Introduction. Let $X^{N}(c)$ denote an $N$-dimensional manifold of constant sectional curvature $c$, and suppose that $M$ is a minimal surface in $X^{N}(c)$ with Riemannian metric $d s^{2}$ and Gauss curvature $K$. The classical theorem of Ricci, as extended by Lawson [3], says that when $N=3$ minimal surfaces of $X^{3}(c)$ are characterized by the conditions that $K \leq c$ and at points where $K<c$ the metric $d \hat{s}^{2}=\sqrt{c-k} d s^{2}$ is flat. Moreover, for each minimal surface $M$ in $X^{3}(c)$, there is a 1-parameter family of isometric minimal surfaces $M_{\tau}, 0 \leq \tau<2 \pi$, such that $M$ is congruent to one of the members of this family.

This paper will describe a class of minimal surfaces in $X^{N}(c)$, called exceptional minimal surfaces, and a sequence of functions $A_{1}^{c}, A_{2}^{c}, \ldots$ on each surface such that when $N=2 n+1$, these surfaces are characterized by the conditions that $A_{r}^{c} \geq 0,1 \leq r \leq n$, and at points where each $A_{r}^{c}>0$, the metric $d \hat{s}^{2}=\left(A_{n}^{c}\right)^{1 /(n+1)} d s^{2}$ is flat. This reduces to the Ricci-Lawson condition when $n=1$, in that $A_{1}^{c}=c-K$. The exceptional minimal surfaces in $X^{2 n+1}(c)$ will be seen to belong to 1-parameter families of isometric surfaces, just as happens in $X^{3}(c)$.

In $X^{2 n+2}(c)$, the exceptional minimal surfaces will be characterized by the conditions that $A_{r}^{c} \geq 0,1 \leq r \leq n$, and $A_{n+1}^{c} \equiv 0$. Additionally, in $X^{2 n+2}(c)$ the exceptional minimal surfaces will be rigid. These results given here for the case where $N=2 n+2$ are actually implicit in [2], although they are stated there in terms of minimal immersions of the 2-sphere $S^{2}$ into $X^{2 n+2}(c)$. 
Sections 1 and 2 summarize the structure equations for minimal surfaces and some results from [2] that are needed here. Section 3 contains the statements of the theorems, which are proved in $\S \S 4$ and 5. Section 6 contains two corollaries on isometric minimal surfaces.

1. Structure equations of surfaces. Suppose $M$ is a Riemannian surface with Gauss curvature $K$. Let $e_{1}, e_{2}$ be a local orthonormal frame field on $M$, and let $\theta_{1}, \theta_{2}$ be the coframe dual to $e_{1}, e_{2}$. Then the structure equations of $M$ are

(1) $d \theta_{1}=\omega_{12} \wedge \theta_{2}, \quad d \theta_{2}=-\omega_{12} \wedge \theta_{2}$, and $d \omega_{12}=-K \theta_{1} \wedge \theta_{2}$,

where $\omega_{12}\left(=-\omega_{21}\right)$ is the connection form on $M$, and $K$ is the Gauss curvature of $M$.

If $f: M \rightarrow \mathbb{R}$ is a smooth function, let $f_{1}, f_{2}$ be given by

$$
d f=f_{1} \theta_{1}+f_{2} \theta_{2} \text {. }
$$

Taking the exterior derivative of this expression and applying Cartan's Lemma gives $f_{11}, f_{12}, f_{21}$, and $f_{22}$, with $f_{12}=f_{21}$, such that

$$
\begin{aligned}
& d f_{1}-f_{2} \omega_{12}=f_{11} \theta_{1}+f_{12} \theta_{2}, \\
& d f_{2}+f_{1} \omega_{12}=f_{21} \theta_{1}+f_{22} \theta_{2} .
\end{aligned}
$$

If $\Delta$ is the Laplace-Beltrami operator of $M$, then $\Delta f=f_{11}+f_{22}$. Let $\bar{\partial} f=\left(f_{1}+i f_{2}\right) / 2$ and $\varphi=\theta_{1}+i \theta_{2}$. Then

$$
\Delta f \varphi \wedge \bar{\varphi}=4\left(d \bar{\partial} f+i \bar{\partial} f \omega_{12}\right) \wedge \bar{\varphi} .
$$

Note that this is not the usual $\bar{\partial}$-operator. If $z=x+i y$ gives local isothermal coordinates on $M$, then there is a positive function $\lambda$ such that $d s^{2}=\lambda^{2}|d z|^{2}$. The $\bar{\partial}$ defined here is $\lambda$ times the usual $\bar{\partial}$-operator.

2. Structure equations and normal planes. Suppose $M$ is a minimal surface in $X^{N}(c)$. When clear from context, this latter manifold will be denoted simply as $X$. Assume that $M$ lies fully in $X$, i.e., does not lie in a totally geodesic submanifold of $X$. Let the integer $n$ be given by $N=2 n+1$ or $2 n+2$, and let indices have the following ranges unless otherwise indicated:

$$
1 \leq j, k \leq 2, \quad 3 \leq \alpha, \beta \leq N, \quad 1 \leq A, B, C \leq N,
$$

$$
1 \leq p, q, r \leq n
$$

(The symbol $i$ will be reserved for $\sqrt{-1}$.) 
Let $\tilde{e}_{A}$ be a local orthonormal frame field on $X$, and let $\tilde{\theta}_{A}$ be the coframe dual to $\tilde{e}_{A}$. Then the structure equations of $X$ are

(3) $d \tilde{\theta}_{A}=\sum_{B} \tilde{\omega}_{A B} \wedge \tilde{\theta}_{B}$ and $d \tilde{\omega}_{A B}=\sum_{C} \tilde{\omega}_{A C} \wedge \tilde{\omega}_{C B}-c \tilde{\theta}_{A} \wedge \tilde{\theta}_{B}$

where the $\tilde{\omega}_{A B} \quad\left(=-\tilde{\omega}_{B A}\right)$ are the connection forms on $X$. If $\langle\cdot, \cdot\rangle$ is the Riemannian metric on $X$, then $\tilde{\omega}_{A B}=\left\langle d \tilde{e}_{A}, \tilde{e}_{B}\right\rangle$.

Suppose that $e_{1}, e_{2}$ is a frame on $M$ as described in the previous section and that the frame $\tilde{e}_{A}$ is chosen so that on $M, e_{j}=\tilde{e}_{j}$, and the $\tilde{e}_{\alpha}$ are normal to $M$. Then differential forms on $M$ can be identified with differential forms on $X$ restricted to $M$ :

$$
\theta_{j}=\left.\tilde{\theta}_{j}\right|_{M} \text { and } \omega_{12}=\left.\tilde{\omega}_{12}\right|_{M} .
$$

To simplify the notation, when forms and vectors on $X$ are restricted to $M$, let them be denoted by the same symbol without tilde:

$$
\begin{aligned}
\theta_{A} & \text { will denote }\left.\tilde{\theta}_{A}\right|_{M}, \\
\omega_{A B} & \text { will denote }\left.\tilde{\omega}_{A B}\right|_{M}, \quad \text { and } \\
e_{A} & \text { will denote }\left.\tilde{e}_{A}\right|_{M} .
\end{aligned}
$$

Then $\theta_{\alpha}=0$ on $M$ since the $e_{\alpha}$ are normal to $M$. When the relation $d \theta_{\alpha}=0$ is expanded using the structure equations (3), Cartan's Lemma can be applied to show that there are functions $h_{\alpha j k}$ such that

$$
\omega_{\alpha j}=\sum_{k} h_{\alpha j k} \theta_{k}, \quad h_{\alpha j k}=h_{\alpha k j} .
$$

The $h_{\alpha j k}$ are the coefficients of the second fundamental form. The assumption that $M$ is a minimal surface is equivalent to assuming that the second fundamental form has zero trace:

$$
h_{\alpha 11}+h_{\alpha 22}=0 \text {. }
$$

Let $T_{x} M$ and $T_{x} X$ denote the tangent space to $M$ and $X$, respectively, at a point $x$. Curves on $M$ through $x$ have their first derivatives at $x$ in $T_{x} M$, but higher order derivatives will have components normal to $M$. The space spanned by the derivatives of order up to $r$ is called the rth osculating space of $M$ at $x$, denoted $T_{x}^{(r)} M$. If for all $r, T_{x}^{(r)} M \varsubsetneqq T_{x} X$ at all $x$ in a neighborhood of $M$, then that neighborhood would lie in a totally geodesic submanifold of $X$ ([4], p. 241). The assumption that $M$ lies fully in $X$ means that for some $r, T_{x}^{(r)} M=T_{x} X$ at generic points of $M$. 
The rth normal space of $M$ at $x$, denoted $\operatorname{Nor}_{x}^{(r)} M$, is the orthogonal complement of $T_{x}^{(r)} M$ in $T_{x}^{(r+1)} M$, so

$$
T_{x}^{(r+1)} M=T_{x}^{(r)} M \oplus \operatorname{Nor}_{x}^{(r)} M \text {. }
$$

The results in $\S 2$ and $\S 3$ of [2] show that at generic points of $M$, the dimension of $\operatorname{Nor}_{x}^{(r)} M$ is 2 when $1 \leq r \leq n-1$, and the dimension of $\operatorname{Nor}_{x}^{(n)} M$ is 1 or 2 , depending on whether $N$ is odd or even. Those normal spaces that have dimension 2 will be called the normal planes of $M$. Let $\beta_{N}$ denote the number of normal planes possessed by $M$ at a generic point:

$$
\beta_{N}= \begin{cases}n, & \text { if } N=2 n+2, \\ n-1, & \text { if } N=2 n+1\end{cases}
$$

Choose the normal vectors $e_{\alpha}$ so that $\operatorname{Nor}_{x}^{(r)} M$ is spanned by $\left\{e_{2 r+1}, e_{2 r+2}\right\}, 1 \leq r \leq \beta_{N}$. When $N=2 n+1$, $\operatorname{Nor}_{x}^{(n)} M$ will be spanned by $\left\{e_{2 n+1}\right\}$. The derivatives of vector fields in $T_{x}^{(r)} M$ must lie in $T_{x}^{(r+1)} M$, so $d e_{2 r-1}$ and $d e_{2 r}$ cannot have any $e_{\alpha}$ components for $\alpha>2 r+2$. Since $\omega_{A B}=\left\langle d e_{A}, e_{B}\right\rangle$ and $\omega_{A B}=-\omega_{B A}$,

$$
\omega_{2 r-1, A}=\omega_{2 r, A}=0 \text { when } A>2 r+2 \text { and when } A<2 r-3 \text {. }
$$

When $r=2$, these relations imply that

$$
h_{\alpha j k}=0 \quad \text { when } \alpha>4 \text {. }
$$

Set $H_{\alpha}=h_{\alpha 11}+i h_{\alpha 12}$ for $\alpha=3$ and 4. Then using (5), (6), and the form $\varphi=\theta_{1}+i \theta_{2}$, equations (4) can be written

$$
\begin{aligned}
\omega_{\alpha 1}+i \omega_{\alpha 2} & =H_{\alpha} \bar{\varphi} \text { for } \alpha=3,4, \\
\omega_{\alpha j} & =0 \text { for } \alpha>4 .
\end{aligned}
$$

Now applying the structure equations (3) to the relation $\omega_{51}+i \omega_{52}=0$ leads to

$$
\left(H_{3} \omega_{53}+H_{4} \omega_{54}\right) \wedge \bar{\varphi}=0
$$

So for some $H_{5}$,

$$
H_{3} \omega_{53}+H_{4} \omega_{54}=H_{5} \bar{\varphi} .
$$

Applying this argument inductively to the relations

$$
\begin{aligned}
& \omega_{2 r+1,2 r-3}+i \omega_{2 r+1,2 r-2}=0, \\
& \omega_{2 r+2,2 r-3}+i \omega_{2 r+2,2 r-2}=0,
\end{aligned}
$$

for $r=2, \ldots, n$, produces $H_{\alpha}$ such that

$$
H_{2 r-1} \omega_{\alpha, 2 r-1}+H_{2 r} \omega_{\alpha, 2 r}=H_{\alpha} \bar{\varphi},
$$


for $\alpha=2 r+1$ and $2 r+2$. This relation extends to the case where $r=1$ by setting $H_{1}=1$, and $H_{2}=i$.

The $r$ th normal plane, $\operatorname{Nor}_{x}^{(r)} M$, of $M$ will be called exceptional if $H_{2 r+2}= \pm i H_{2 r+1}$. (Note that the sign can be reversed by reversing the orientation of $\operatorname{Nor}_{x}^{(r)} M$. Note also that when $N=2 n+1, \operatorname{Nor}_{x}^{(n)} M$ is a line, not a plane, and the notion of exceptionality does not apply.) The minimal surface $M$ will be called exceptional if all of its normal planes are exceptional. Minimal immersions of the 2-sphere $S^{2}$ into $X^{2 n+2}(c)$ are always exceptional [2]. (These surfaces are called "superminimal" by Bryant [1].)

For the remainder of this paper, assume that $M$ is an exceptional minimal surface. The orientations of the normal planes of such a surface can be chosen so that $H_{2 r+2}=+i H_{2 r+1}$ for $1 \leq r \leq \beta_{N}$. Then equations (7) become

$$
\begin{aligned}
& H_{2 r-1}\left(\omega_{2 r+1,2 r-1}+i \omega_{2 r+1,2 r}\right)=H_{2 r+1} \bar{\varphi}, \\
& H_{2 r-1}\left(\omega_{2 r+2,2 r-1}+i \omega_{2 r+2,2 r}\right)=i H_{2 r+1} \bar{\varphi},
\end{aligned}
$$

for $r=1, \ldots, \beta_{N}$, together with

$$
H_{2 n-1}\left(\omega_{2 n+1,2 n-1}+i \omega_{2 n+1,2 n}\right)=H_{2 n+1} \bar{\varphi}
$$

when $N=2 n+1$.

3. The theorems. For each real number $c$, the quantities $A_{p}^{c}$ are defined as follows:

$$
\begin{aligned}
A_{0}^{c} & =1 / 2 \text { for all } c, \\
A_{1}^{c} & =c-K, \\
A_{p+1}^{c} & = \begin{cases}A_{p}^{c}\left[\Delta \log \left(A_{p}^{c}\right)+A_{p}^{c} / A_{p-1}^{c}-2(p+1) K\right], & \text { if } A_{p}^{c}>0, \\
0, & \text { otherwise. }\end{cases}
\end{aligned}
$$

Theorem A. Suppose $M$ is an exceptional minimal surface lying fully in $X^{N}(c)$. Let $K$ denote the Gauss curvature of $M, d s^{2}$ its Riemannian metric, and let $n$ be given by $N=2 n+1$ or $2 n+2$. Then $A_{p}^{c} \geq 0$ for $1 \leq p \leq n$, with equality only at isolated points. If $N=2 n+2$, then $A_{n+1}^{c} \equiv 0$, and if $N=2 n+1$, then at points where each $A_{p}^{c}>0$, the metric $d \hat{s}^{2}=\left(A_{n}^{c}\right)^{1 /(n+1)} d s^{2}$ is flat (has Gauss curvature $\hat{K} \equiv 0)$.

Theorem B. Suppose $M$ is a smooth Riemannian surface with Gauss curvature $K$ and Riemannian metric $d s^{2}$. Suppose $A_{p}^{c}>0$ for $1 \leq p \leq n$ in some neighborhood of $x_{0} \in M$. If $A_{n=1}^{c} \equiv 0$, set 
$N=2 n+2$. If the metric $d \hat{s}^{2}=\left(A_{n}^{c}\right)^{1 /(n+1)} d s^{2}$ is flat, set $N=2 n+1$. Then there is a neighborhood $U$ of $x_{0}$ and an isometric immersion $f: U \rightarrow X^{N}(c)$ such that $f(U)$ is an exceptional minimal surface lying fully in $X^{N}(c)$.

The theorems will be proved in $\S 4$ and $\S 5$. Both proofs will use the following:

LEMMA. The condition that $d \hat{s}^{2}=\left(A_{n}^{c}\right)^{1 /(n+1)} d s^{2}$ should be flat is equivalent to

$$
\Delta \log \left(A_{n}^{c}\right)-2(n+1) K \equiv 0 .
$$

Proof. Recall that if $d s^{2}=\lambda^{2}|d z|^{2}$ for isothermal coordinates $z=$ $x+i y$, then the Gauss curvature is given by $K=-\Delta \log \lambda$. If $d \hat{s}^{2}=$ $\left(A_{n}^{c}\right)^{1 /(n+1)} d s^{2}=\lambda^{2}\left(A_{n}^{c}\right)^{1 /(n+1)}|d z|^{2}$, then $\hat{K}=K-\left[\Delta \log \left(A_{n}^{c}\right)\right] / 2(n+1)$ so $\hat{K} \equiv 0$ if and only if (10) holds.

\section{Proof of Theorem $A$.}

LEMMA 4.1. If $M \subset X^{N}(c)$ is an exceptional minimal surface, then (11) $d \omega_{2 r-1,2 r}=2\left[\left|H_{2 r+1}\right|^{2} /\left|H_{2 r-1}\right|^{2}-\left|H_{2 r-1}\right|^{2} /\left|H_{2 r-3}\right|^{2}\right] \theta_{1} \wedge \theta_{2}$ for $r=2, \ldots, \beta_{N}$, whenever the denominators are not zero.

Proof. From the first equation in (8),

$$
\begin{aligned}
& H_{2 r-1}\left(\omega_{2 r+1,2 r-1}+i \omega_{2 r+1,2 r}\right) \wedge \bar{H}_{2 r-1}\left(\omega_{2 r+1,2 r-1}-i \omega_{2 r+1,2 r}\right) \\
& \quad=-H_{2 r+1} \bar{H}_{2 r+1} \varphi \wedge \bar{\varphi} .
\end{aligned}
$$

Since $\varphi \wedge \bar{\varphi}=-2 i \theta_{1} \wedge \theta_{2}$, this implies that

$$
\omega_{2 r-1,2 r+1} \wedge \omega_{2 r+1,2 r}=\left[\left|H_{2 r+1}\right|^{2} /\left|H_{2 r-1}\right|^{2}\right] \theta_{1} \wedge \theta_{2} .
$$

Similarly,

$$
\omega_{2 r-1,2 r+2} \wedge \omega_{2 r+2,2 r}=\left[\left|H_{2 r+1}\right|^{2} /\left|H_{2 r-1}\right|^{2}\right] \theta_{1} \wedge \theta_{2} .
$$

From (8) with $r$ replaced by $r-1$,

$$
\begin{gathered}
\bar{H}_{2 r-3}\left(\omega_{2 r-1,2 r-3}-i \omega_{2 r-1,2 r-2}\right) \wedge H_{2 r-3}\left(\omega_{2 r, 2 r-3}+i \omega_{2 r, 2 r-2}\right) \\
=\bar{H}_{2 r-1} \varphi \wedge i H_{2 r-1} \bar{\varphi} .
\end{gathered}
$$

The real part of this expression simplifies to

$$
\begin{gathered}
\omega_{2 r-1,2 r-3} \wedge \omega_{2 r-3,2 r}+\omega_{2 r-1,2 r-2} \wedge \omega_{2 r-2,2 r} \\
\quad=-2\left[\left|H_{2 r-1}\right|^{2} /\left|H_{2 r-3}\right|^{2}\right] \theta_{1} \wedge \theta_{2} .
\end{gathered}
$$

Now (11) follows from the structure equation (3) for $d \omega_{2 r-1,2 r}$ and from (12), (13), and (14). 
LEMMA 4.2. For each $r=1, \ldots, \beta_{N}+1$, there is a $G_{2 r-1}$ such that

$$
d H_{2 r-1}+i H_{2 r-1}\left(r \omega_{12}-\omega_{2 r-1,2 r}\right)=G_{2 r-1} \bar{\varphi} .
$$

Proof. When $r=1, H_{1}=1$, so $G_{1}=0$. Suppose (15) holds when $r=p$. Set $r=p$ in the two equations in (8) and take their exterior derivatives using the structure equations (3). (Note that $d \bar{\varphi}=$ $i \omega_{12} \wedge \bar{\varphi}$.) In both cases, the result is

$$
\left\{d H_{2 p+1}+i H_{2 p+1}\left[(p+1) \omega_{12}-\omega_{2 p+1,2 p+2}\right]\right\} \wedge \bar{\varphi}=0,
$$

so for some $G_{2 p+1}$,

$$
d H_{2 p+1}+i H_{2 p+1}\left[(p+1) \omega_{12}-\omega_{2 p+1,2 p+2}\right]=G_{2 p+1} \bar{\varphi}
$$

and the lemma follows by induction.

COROLlARY. If $\mathrm{H}_{2 r-1}$ is not identically zero, then its zeros are isolated.

Proof. By (15),

$$
d \bar{H}_{2 r-1}-i \bar{H}_{2 r-1}\left(r \omega_{12}-\omega_{2 r-1,2 r}\right) \equiv 0 \quad(\bmod \varphi),
$$

and the corollary follows from the theorem in $\S 4$ in [2].

LEMMA 4.3. If $M \subset X^{N}(c)$ is an exceptional minimal surface, then

$$
A_{r}^{c}=2^{2 r-1}\left|H_{2 r+1}\right|^{2}, \quad r=0, \ldots, \beta_{N},
$$

and the zeros of each $A_{r}^{c}$ are isolated.

Proof. The preceding corollary shows that the zeros of $A_{r}^{c}$ are isolated whenever (16) holds. Clearly (16) holds when $r=0$. To show (16) when $r=1$, note that the structure equations (1) and (3) give two ways of computing $d \omega_{12}$ :

$$
d \omega_{12}=-K \theta_{1} \wedge \theta_{2}=\sum_{\alpha=3}^{4} \omega_{1 \lambda} \wedge \omega_{\alpha 2}-c \theta_{1} \wedge \theta_{2} .
$$

Expanding the summation using equations (8) with $r=1$ and then extracting the coefficients of $\theta_{1} \wedge \theta_{2}$ yields

$$
A_{1}^{c}=c-K=2\left|H_{3}\right|^{2} \text {. }
$$

Now proceed inductively: suppose that for some $p,(16)$ holds for $r<p$. If $A_{p-1}^{c}=0$, then $A_{p}^{c}=0$ by definition. Also, $H_{2 p-1}=0$ by (16), so $H_{2 p+1}=0$ by (8), showing that (16) holds for $r=p$. Since 
the zeros of the $A$ 's propagate along the sequence, the only other case to consider is $A_{p-2}^{c} \neq 0$ and $A_{p-1}^{c} \neq 0$. By Lemma 4.1,

$$
d \omega_{2 p-1,2 p}=\frac{1}{2}\left[2^{2 p-1}\left|H_{2 p+1}\right|^{2} / A_{p-1}^{c}-A_{p-1}^{c} / A_{p-2}^{c}\right] \theta_{1} \wedge \theta_{2} .
$$

Using this, the exterior derivative of (15) when $r=p$ is

$$
\begin{aligned}
& \left\{d G_{2 p-1}+i G_{2 p-1}\left[(p+1) \omega_{12}-\omega_{2 p-1,2 p}\right]\right\} \wedge \bar{\varphi} \\
& =-\frac{i}{2} H_{2 p-1}\left[2^{2 p-1}\left|H_{2 p+1}\right|^{2} / A_{p-1}^{c}\right. \\
& \left.\quad-A_{p-1}^{c} / A_{p-2}^{c}+2 p K\right] \theta_{1} \wedge \theta_{2} .
\end{aligned}
$$

By Lemma 4.2, the exterior derivative of $A_{p-1}^{c}=2^{2 p-3} H_{2 p-1} \bar{H}_{2 p-1}$ is

$$
\left(A_{p-1}^{c}\right)_{1} \theta_{1}+\left(A_{p-1}^{c}\right)_{2} \theta_{2}=2^{2 p-3}\left(H_{2 p-1} \bar{G}_{2 p-1} \varphi+\bar{H}_{2 p-1} G_{2 p-1} \bar{\varphi}\right) .
$$

Wedging this with $\varphi$ and comparing coefficients of $i \theta_{1} \wedge \theta_{2}$ yields

$$
\bar{\partial} A_{p-1}^{c}=2^{2 p-3} \bar{H}_{2 p-1} G_{2 p-1} .
$$

By (2), (15), and (19),

$$
\begin{aligned}
\Delta A_{p-1}^{c} \varphi \wedge \bar{\varphi}= & 2^{2 p-1} \bar{H}_{2 p-1} \\
& \times\left\{d G_{2 p-1}+i G_{2 p-1}\left[(p+1) \omega_{12}-\omega_{2 p-1,2 p}\right]\right\} \\
& \wedge \bar{\varphi}+2^{2 p-1}\left|G_{2 p-1}\right|^{2} \varphi \wedge \bar{\varphi} .
\end{aligned}
$$

Note that by (19) and the inductive hypothesis,

$$
\left|G_{2 p-1}\right|^{2}=\left|\bar{\partial} A_{p-1}^{c}\right|^{2} / 2^{4 p-6}\left|H_{2 p-1}\right|^{2}=\left\|d A_{p-1}^{c}\right\|^{2} / 2^{2 p-1} A_{p-1}^{c} .
$$

Combining this with (20) and (18) shows that

$$
\begin{aligned}
\Delta A_{p-1}^{c}= & A_{p-1}^{c}\left[2^{2 p-1}\left|H_{2 p+1}\right|^{2} / A_{p-1}^{c}-A_{p-1}^{c} / A_{p-2}^{c}+2 p K\right] \\
& +\left\|d A_{p-1}^{c}\right\|^{2} / 2 A_{p-1}^{c} .
\end{aligned}
$$

Thus,

$$
\begin{aligned}
2^{2 p-1}\left|H_{2 p+1}\right|^{2} & =A_{p-1}^{c}\left[\Delta \log \left(A_{p-1}^{c}\right)+A_{p-1}^{c} / A_{p-2}^{c}-2 p K\right] \\
& =A_{p}^{c},
\end{aligned}
$$

completing the induction.

To finish the proof of Theorem A, consider first the case $N=2 n+2$. Calculations analogous to those in the proof of Lemma 4.1 show that $d \omega_{2 n+1,2 n+2}=-2\left[\left|H_{2 n+1}\right|^{2} /\left|H_{2 n-1}\right|^{2}\right] \theta_{1} \wedge \theta_{2}=-\left[A_{n}^{c} / 2 A_{n-1}^{c}\right] \theta_{1} \wedge \theta_{2}$. 
Using this in the proof of Lemma 4.3 with $p=n+1$ shows that

$$
A_{n+1}^{c}=A_{n}^{c}\left[\Delta \log \left(A_{n}^{c}\right)+A_{n}^{c} / A_{n-1}^{c}-2(n+1) K\right] \equiv 0 .
$$

The proof of Theorem A when $N=2 n+1$ follows by modifying the arguments in the proofs of the lemmas to apply to (9) instead of (8). Using (9) in the proof of Lemma 4.1 when $r=n$ yields

$$
d \omega_{2 n-1,2 n}=2\left[\left|H_{2 n+1}\right|^{2} / 2\left|H_{2 n-1}\right|^{2}-\left|H_{2 n-1}\right|^{2} /\left|H_{2 n-3}\right|^{2}\right] \theta_{1} \wedge \theta_{2} .
$$

The proof of Lemma 4.2 applied to (9) implies that for some $G_{2 n+1}$,

$$
d H_{2 n+1}+i H_{2 n+1}(n+1) \omega_{12}=G_{2 n+1} \bar{\varphi} .
$$

The proof of Lemma 4.3 when $p=n$ shows that

$$
A_{n}^{c}=2^{2 n-2}\left|H_{2 n+1}\right|^{2}
$$

so that

$$
d \omega_{2 n-1,2 n}=\frac{1}{2}\left[A_{n}^{c} / A_{n-1}^{c}-A_{n-1}^{c} / A_{n-2}^{c}\right] \theta_{1} \wedge \theta_{2} .
$$

Using this, the proof of Lemma 4.3 can be repeated again with appropriate modifications when $p=n+1$ to show that $\Delta \log \left(A_{n}^{c}\right)-$ $2(n+1) K=0$. By the lemma in $\S 3, d \hat{s}^{2}=\left(A_{n}^{c}\right)^{1 /(n+1)} d s^{2}$ is flat.

5. Proof of Theorem B. Let $F(M)$ and $F(X)$ denote the bundles of orthonormal frames on $M$ and $X$, respectively. Consider the manifold

$$
P=F(M) \times F(X) \times \mathbb{C}^{2 n}
$$

where $\mathbb{C}^{2 n}$ has coordinates $\left(H_{3}, \ldots, H_{2 n+1}, G_{3}, \ldots, G_{2 n+1}\right)$. Use the projections $\pi_{1}: P \rightarrow F(M)$ and $\pi_{2}: P \rightarrow F(X)$ to pull the forms $\theta_{j}, \omega_{12}, \tilde{\theta}_{A}$, and $\tilde{\omega}_{A B}$ back to $P$, and let the pulled-back forms be denoted by the same symbols. For example, the pull-back $\pi_{1}^{*}\left(\theta_{j}\right)$ will be denoted simply $\theta_{j}$. Let $I$ denote the ideal of differential forms on $P$ generated by the following 1 -forms:

$$
\begin{array}{r}
\tilde{\theta}_{j}-\theta_{j}, \quad \tilde{\theta}_{\alpha}, \quad \tilde{\omega}_{12}-\omega_{12}, \\
\tilde{\omega}_{2 r-1, A} \text { and } \tilde{\omega}_{2 r, A} \quad \text { for } 1 \leq r \leq \beta_{N}, \\
\quad \text { and } A>2 r+2 \text { or } A<2 r-3, \\
H_{2 r-1}\left(\tilde{\omega}_{2 r+1,2 r-1}+i \tilde{\omega}_{2 r+1,2 r}\right)-H_{2 r+1} \bar{\varphi}, \quad 1 \leq r \leq \beta_{N}, \\
H_{2 r-1}\left(\tilde{\omega}_{2 r+2,2 r-1}+i \tilde{\omega}_{2 r+2,2 r}\right)-i H_{2 r+1} \bar{\varphi}, \quad 1 \leq r \leq \beta_{N}, \\
d H_{2 r+1}+i H_{2 r+1}\left[(r+1) \omega_{12}-\tilde{\omega}_{2 r+1,2 r+2}\right]-G_{2 r+1} \bar{\varphi}, \\
0 \leq r \leq \beta_{N},
\end{array}
$$


together with the forms

$$
\begin{aligned}
& H_{2 n-1}\left(\tilde{\omega}_{2 n+1,2 n-1}+i \tilde{\omega}_{2 n+1,2 n}\right)-H_{2 n+1} \bar{\varphi}, \\
& d H_{2 n+1}+i H_{2 n+1}(n+1) \omega_{12}-G_{2 n+1} \bar{\varphi},
\end{aligned}
$$

when $N=2 n+1$. Let $Q$ be the submanifold of $P$ determined by the relations

$$
\begin{array}{r}
A_{r}^{c}=2^{2 r-1}\left|H_{2 r+1}\right|^{2} \text { and } \bar{\partial} A_{r}^{c}=2^{2 r-1} \bar{H}_{2 r+1} G_{2 r+1} \\
\text { for } 1 \leq r \leq \beta_{N},
\end{array}
$$

together with

$$
A_{n}^{c}=2^{2 n-2}\left|H_{2 n+1}\right|^{2} \text { and } \bar{\partial} A_{n}^{c}=2^{2 n-2} \bar{H}_{2 n+1} G_{2 n+1}
$$

when $N=2 n+1$. Lengthy calculations (most of which are outlined in $\S 2$ and $\S 4$ ) show that if $N=2 n+2$ and $A_{n+1}^{c} \equiv 0$, or if $N=2 n+1$ and $\Delta \log \left(A_{n}^{c}\right)-2(n+1) K \equiv 0$, then $I$ is a closed ideal in $Q$, i.e., if $\eta \in I$, then $d \eta \in I$. Choose an initial point of $Q$ by choosing initial points $x_{0} \in M, y_{0} \in X$, initial frames $e_{i}$ and $\tilde{e}_{A}$, and initial values of the $H$ 's and $G$ 's that satisfy (21) and (22) at $x_{0}$. By the Frobenius Theorem, there is a submanifold $S \subset Q$ passing through this initial point such that all forms in $I$ are zero on $S$.

When $N=2 n+2, \operatorname{dim} Q=2 n^{2}+6 n+6$ and there are $2 n^{2}+6 n+3$ independent 1 -forms in $I$, so $\operatorname{dim} S=3$. By a standard argument ([5], pp. 73-77) the restricted projection $\left.\pi_{1}\right|_{S}$ is locally a diffeomorphism from $S$ to $F(M)$. More precisely, there is a neighborhood $V \subset S$ and a neighborhood $W \subset F(M)$ containing $\left(x_{0}, e_{1}, e_{2}\right)$ such that $\left.\pi_{1}\right|_{V}: V \rightarrow W$ is a diffeomorphism. Define $f_{*}: W \rightarrow F(X)$ by

$$
f_{*}=\pi_{2} \circ\left(\left.\pi_{1}\right|_{V}\right)^{-1} \text {. }
$$

Let $f_{*}\left(x, e_{1}, e_{2}\right)=\left(y, \tilde{e}_{1}, \tilde{e}_{2}, \ldots, \tilde{e}_{2 n+2}\right)$ for $\left(x, e_{1}, e_{2}\right) \in W$. If $f_{*}$ is a bundle map, then it projects down to a map $f: U \rightarrow X$ where $U \subset M$ is a neighborhood of $x_{0}$. The forms in $I$ were chosen so that $f$ would be an isometry $\left(\theta_{i}=f^{*}\left(\tilde{\theta}_{i}\right)\right.$, where $f^{*}$ is the pull-back induced by $f$ ) and so that $f(U)$ would be an exceptional minimal surface. Note that since $A_{r}^{c}>0,1 \leq r \leq n$, each $H_{2 r+1} \neq 0$ by (21), so $f(U)$ lies fully in $X^{2 n+2}(c)$.

To show that $f_{*}$ is a bundle map, let $a$ be a complex number with absolute value 1 and consider the action on $P$ given by

$$
\begin{aligned}
e_{1}+i e_{2} & \rightarrow a\left(e_{1}+i e_{2}\right), \\
\tilde{e}_{2 r-1}+i \tilde{e}_{2 r} & \rightarrow a^{r}\left(\tilde{e}_{2 r-1}+i \tilde{e}_{2 r}\right), \quad 1 \leq r \leq n+1, \\
G_{2 r+1} & \rightarrow a G_{2 r+1}, \quad 1 \leq r \leq n,
\end{aligned}
$$


with $x \in M, y \in X$, and the $H$ 's unchanged. This induces the following action on forms:

$$
\begin{aligned}
\theta_{1}+i \theta_{2} & \rightarrow a\left(\theta_{1}+i \theta_{2}\right), \\
\tilde{\theta}_{2 r-1}+i \tilde{\theta}_{2 r} & \rightarrow a^{r}\left(\tilde{\theta}_{2 r-1}+i \tilde{\theta}_{2 r}\right), \quad 1 \leq r \leq n+1, \\
\eta_{p, q} & \rightarrow a^{p+q} \eta_{p, q}, \quad 1 \leq p, q \leq n+1,
\end{aligned}
$$

where

$$
\eta_{p, q}=\tilde{\omega}_{2 p-1,2 q-1}+i \tilde{\omega}_{2 p-1,2 q}+i\left(\tilde{\omega}_{2 p, 2 q-1}+i \tilde{\omega}_{2 p, 2 q}\right) .
$$

The forms $\omega_{12}$ and $\tilde{\omega}_{2 r+1,2 r+2}$ are unchanged. Also,

$$
\bar{\partial} \rightarrow a \bar{\partial} \text {. }
$$

The submanifold $Q$ and the ideal $I$ are invariant under this action, so the integral submanifolds of $I$ are also invariant. It follows that $f_{*}$ is a bundle map, which completes the proof when $N=2 n+2$.

When $N=2 n+1, \operatorname{dim} Q=2 n^{2}+4 n+4$ and there are $2 n^{2}+4 n+2$ independent 1 -forms in $I$, so $\operatorname{dim} S=2$. The initial conditions are such that $\theta_{1} \wedge \theta_{2} \neq 0$ at the initial point and therefore in a neighborhood of the initial point. Thus, there are neighborhoods $V \subset S$ and $U \subset M$ such that projection from $S$ to $M$ is a diffeomorphism from $V$ to $U$, and $f$ can be defined as the inverse of this projection followed by the projection from $S$ to $X$. As in the previous case, $f$ is an isometry and $f(U)$ is an exceptional minimal surface lying fully in $X^{2 n+1}(c)$.

\section{Isometric exceptional minimal surfaces.}

Corollary 6.1. Suppose $M_{1}$ and $M_{2}$ are exceptional minimal surfaces lying fully in $X^{2 n+2}(c)$. If $M_{1}$ and $M_{2}$ are isometric, then they are congruent.

Proof. The surfaces $M_{1}$ and $M_{2}$ are real analytic, so it suffices to show that they are locally congruent. By Theorem $\mathrm{A}, A_{n+1}^{c} \equiv 0$ and there are isometric neighborhoods $U_{1}, U_{2}$ in $M_{1}, M_{2}$, respectively, on which $A_{p}^{c}>0,1 \leq p \leq n$. Let $g: U_{1} \rightarrow U_{2}$ be the isometry and let $\tilde{e}_{A}^{j}$ denote frame fields on $X$ adapted to $U_{j}$ such that $\tilde{e}_{k}^{2}=$ $g_{*}\left(\tilde{e}_{k}^{1}\right)$. These frames determine $H_{2 r+1}^{j}$ and $G_{2 r+1}^{j}$ as in $\S 2$ and $\S 4$, and thus determine submanifolds of $P$ that are integral manifolds for the ideal $I$. Since that ideal satisfies the Frobenius condition, its integral manifolds are completely determined by initial conditions. Let $x_{1} \in$ $U_{1}$ and $x_{2}=g\left(x_{1}\right) \in U_{2}$ be the initial points. The $H$ 's and $G$ 's 
satisfy (21), so for each $r$ there is a value of $\tau$ such that at the initial points, $H_{2 r+1}^{2}=e^{i \tau} H_{2 r+1}^{1}$ and $G_{2 r+1}^{2}=e^{i \tau} G_{2 r+1}^{1}$. By (8), rotating the vectors $\tilde{e}_{2 r+1}^{1}, \tilde{e}_{2 r+2}^{2}$ counterclockwise in $\operatorname{Nor}_{x_{1}}^{(r)} M_{1}$ through an angle $-\tau$ changes $H_{2 r+1}^{1}$ to $e^{-i \tau} H_{2 r+1}^{1}$. By (15), this rotation changes $G_{2 r+1}^{1}$ to $e^{-i \tau} G_{2 r+1}^{1}$. It follows that the normal vectors $\tilde{e}_{\alpha}^{1}$ can be chosen so that $H_{2 r+1}^{1}=H_{2 r+1}^{2}$ and $G_{2 r+1}^{1}=G_{2 r+1}^{2}$ at the initial points. With this choice of the frame $\tilde{e}_{A}^{1}$, an isometry of $X$ that takes $x_{1}$ to $x_{2}$ and $\tilde{e}_{A}^{1}$ and $\tilde{e}_{A}^{2}$ will take $U_{1}$ to $U_{2}$ and therefore $M_{1}$ to $M_{2}$.

COROLlary 6.2. Suppose $M$ is an exceptional minimal surface lying fully in $X^{2 n+1}(c)$. Then there is a 1-parameter family $M_{\tau}$, $0 \leq \tau<2 \pi$, of exceptional minimal surfaces in $X^{2 n+1}(c)$ such that every exceptional minimal surface in $X^{2 n+1}(c)$ that is isometric to $M$ is congruent to some $M_{\tau}$.

Proof. As in the proof of the previous corollary, different exceptional minimal surfaces in $X^{2 n+1}(c)$ that are isometric to $M$ can only arise through a choice of different initial conditions, and choosing different initial values for $H_{3}, H_{5}, \ldots, H_{2 n-1}, G_{3}, G_{5}, \ldots, G_{2 n-1}$ is equivalent to choosing different initial normal vectors $\tilde{e}_{3}, \ldots, \tilde{e}_{2 n}$. As for $H_{2 n+1}$ and $G_{2 n+1}$, they can be replaced by $e^{i \tau} H_{2 n+1}$ and $e^{i \tau} G_{2 n+1}$ in (22), but this is not equivalent to a rotation in $\operatorname{Nor}_{x}^{(n)} M$, which is 1-dimensional. Thus, an integral manifold for the ideal $I$ is determined by initial points in $M$ and $X^{2 n+1}(c)$, initial frames $e_{j}$ and $\tilde{e}_{A}$, and one value of $\tau$ to determine the initial values $e^{i \tau} H_{2 n+1}$ and $e^{i \tau} G_{2 n+1}$. Varying $\tau$ will produce a 1-parameter family $M_{\tau}$ of minimal surfaces and every exceptional minimal surface in $X^{2 n+1}(c)$ that is isometric to $M$ will be congruent to a member of this family.

Added in proof. When $N$ is even, my exceptional minimal surfaces are also known as isotopic minimal surfaces. See [6] for an alternate definition in this case.

\section{REFERENCES}

[1] R. L. Bryant, Conformal and minimal immersions of compact surfaces into the 4-sphere, J. Differential Geom., 17, no. 3, (1982), 455-473.

[2] S.-S. Chern, On the minimal immersions of the two-sphere in a space of constant curvature, Problems in Analysis, Princeton Univ. Press, (1970), 27-40.

[3] H. B. Lawson, Complete minimal surfaces in $S^{3}$, Ann. of Math., 92 (1970), 335-374. 
[4] M. Spivak, A Comprehensive Introduction to Differential Geometry, vol. 4, Publish or Perish, 1975.

[5] F. W. Warner, Foundations of Differentiable Manifolds and Lie Groups, Scott, Foresman, 1971.

[6] H. B. Lawson, Surfaces minimales et la construction de Calabi-Penrose, Séminaire Bourbaki, 1983/84, Astérisque, 121-122 (1985), 197-211.

Received October 18, 1989.

Franklin and Marshall College

LANCASTER, PA 17604 



\section{PACIFIC JOURNAL OF MATHEMATICS EDITORS}

\author{
V. S. VARADARAJAN \\ (Managing Editor) \\ University of California \\ Los Angeles, CA 90024-1555-05 \\ Herbert Clemens \\ University of Utah \\ Salt Lake City, UT 84112 \\ THOMAS ENRIGHT \\ University of California, San Diego \\ La Jolla, CA 92093
}

R. FINN

Stanford University

Stanford, CA 94305

HeRmann FlaschKa

University of Arizona

Tucson, AZ 85721

VAUGHAN F. R. JoNES

University of California

Berkeley, CA 94720

STEVEN KERCKHOFF

Stanford University

Stanford, CA 94305
C. C. MOORE

University of California

Berkeley, CA 94720

MaRTIN SCHARLEMANN

University of California

Santa Barbara, CA 93106

HAROLD STARK

University of California, San Diego

La Jolla, CA 92093

\section{ASSOCIATE EDITORS}
R. ARENS
E. F. BECKENBACH
B. H. NeumanN
F. WolF
K. YosHIDA

\section{SUPPORTING INSTITUTIONS}

UNIVERSITY OF ARIZONA

UNIVERSITY OF BRITISH COLUMBIA

CALIFORNIA INSTITUTE OF TECHNOLOGY

UNIVERSITY OF CALIFORNIA

MONTANA STATE UNIVERSITY

UNIVERSITY OF NEVADA, RENO

NEW MEXICO STATE UNIVERSITY

OREGON STATE UNIVERSITY
UNIVERSITY OF OREGON

UNIVERSITY OF SOUTHERN CALIFORNIA

STANFORD UNIVERSITY

UNIVERSITY OF HAWAII

UNIVERSITY OF TOKYO

UNIVERSITY OF UTAH

WASHINGTON STATE UNIVERSITY

UNIVERSITY OF WASHINGTON 


\section{Pacific Journal of Mathematics}

Vol. 149, No. $1 \quad$ May, 1991

Takao Akahori and Harunori Ameku, On the Romanov kernel and Kuranishi's $L^{2}$-estimate for $\bar{\partial}_{\mathrm{b}}$ over a ball in the strongly pseudo convex

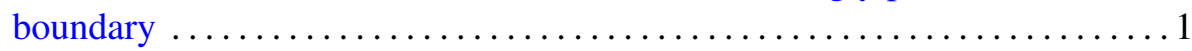

Robert Emile Beaudoin, The proper forcing axiom and stationary set

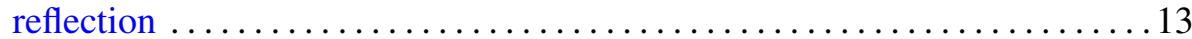

Hans Engler, A matrix Volterra integrodifferential equation occurring in

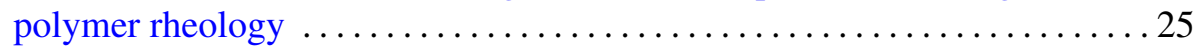

Zhong Ge, On a constrained variational problem and the spaces of

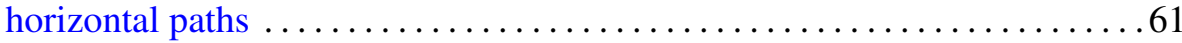

Yutaka Hemmi, Higher homotopy commutativity of $H$-spaces and the

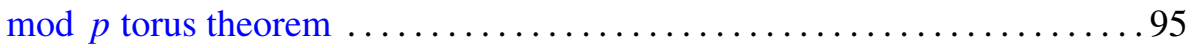

G. D. Johnson, An intrinsic characterization of a class of minimal surfaces

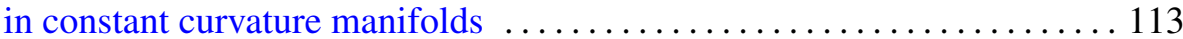

Min Ho Lee, Conjugates of equivariant holomorphic maps of symmetric domains

Jacek Nikiel, H. Murat Tuncali and Edward D. Tymchatyn, On the rim-structure of continuous images of ordered compacta

Tara Lynn Smith, Generalized Clifford-Littlewood-Eckmann groups . . . . . 157

Tara Lynn Smith, Generalized Clifford-Littlewood-Eckmann groups II:

Linear representations and applications 185 